ARTICLE

Received 9 May 2014 | Accepted 27 Jun 2014 | Published 1 Aug 2014

DOI: $10.1038 /$ ncomms5551

\title{
Anisotropic thermal conductivity in uranium dioxide
}

\author{
K. Gofryk ${ }^{1, \dagger}$, S. Du ${ }^{2 \dagger}$, C.R. Stanek ${ }^{3}$, J.C. Lashley ${ }^{1}$, X.-Y. Liu ${ }^{3}$, R.K. Schulze ${ }^{1}$, J.L. Smith ${ }^{1}$, D.J. Safarik ${ }^{1}$ \\ D.D. Byler ${ }^{3}$, K.J. McClellan ${ }^{3}$, B.P. Uberuaga ${ }^{3}$, B.L. Scott ${ }^{4} \&$ D.A. Andersson ${ }^{3}$
}

The thermal conductivity of uranium dioxide has been studied for over half a century, as uranium dioxide is the fuel used in a majority of operating nuclear reactors and thermal conductivity controls the conversion of heat produced by fission events to electricity. Because uranium dioxide is a cubic compound and thermal conductivity is a second-rank tensor, it has always been assumed to be isotropic. We report thermal conductivity measurements on oriented uranium dioxide single crystals that show anisotropy from $4 \mathrm{~K}$ to above $300 \mathrm{~K}$. Our results indicate that phonon-spin scattering is important for understanding the general thermal conductivity behaviour, and also explains the anisotropy by coupling to the applied temperature gradient and breaking cubic symmetry.

\footnotetext{
${ }^{1}$ Materials Technology-Metallurgy, Materials Science and Technology Division, Los Alamos National Laboratory, Los Alamos, New Mexico 87545, USA. ${ }^{2}$ Physics and Chemistry of Materials, Theoretical Division, Los Alamos National Laboratory, Los Alamos, New Mexico 87545, USA. ${ }^{3}$ Materials Science in Radiation \& Dynamical Extremes, Materials Science and Technology Division, Los Alamos National Laboratory, Los Alamos, New Mexico 87545, USA.

${ }^{4}$ Materials Physics and Applications Division, Los Alamos National Laboratory, Los Alamos, New Mexico 87545, USA. † Present address: Fuel Modeling and Simulation Department, Idaho National Laboratory, P.O. Box 1625, Idaho Falls, Idaho 83415, USA (K.G.); Present address: Division of Functional Materials and Nanodevices, Ningbo Institute of Materials Technology and Engineering, Chinese Academy of Sciences, Ningbo, Zhejiang 315201, China (S.D.).

Correspondence and requests for materials should be addressed to C.R.S. (email: stanek@lanl.gov) or to D.A.A. (email: andersson@lanl.gov).
} 
$\mathrm{T}$ oday nearly $20 \%$ of the world's electricity is generated by nuclear energy from $\mathrm{UO}_{2}$ fuel. The thermal conductivity of $\mathrm{UO}_{2}$ governs the conversion of heat produced from fission events into electricity and it is an important parameter in reactor design and safety. Scatter among thermal conductivity $(\kappa)$ data from previous experimental studies on $\mathrm{UO}_{2}$ single crystals cannot easily be explained by sample impurities or inherent measurement errors. (see ref. 1 and references therein). We hypothesize that much of this scatter is due to hidden anisotropy, overlooked in previous experiments that did not specify single crystal orientation. The crystal symmetry of materials dictates the symmetry of the response to applied potential gradients. Thermal conductivity is a second-rank tensor (that is, $\kappa=\kappa_{i j}$ ) that governs the flow of energy under an applied temperature gradient. For materials with cubic point and space group symmetry it is isotropic. Thermal conductivity is almost exclusively dominated by phonons in insulators and semiconductors. At very low temperatures, where ballistic phonon transport may occur, exceptions to the isotropy of cubic materials have been reported, for example, ref. 2. This can be explained by the thermal conductivity being a fourth rank tensor $\left(\kappa=\kappa_{i j l m}\right)$ related to the elastic stiffness tensor when phonon scattering is minimal ${ }^{3}$.

Here we show by experiments on single crystals that $\mathrm{UO}_{2}$, which crystallizes in the cubic fluorite structure $(\mathrm{Fm} \overline{\mathrm{3}} \mathrm{m})$, exhibits anisotropic thermal conductivity at temperatures at which ballistic mechanisms are not possible. The anisotropy is rather due to the applied temperature gradient interacting with the electric moments on uranium ions thus affecting the phononspin interactions to slightly break cubic symmetry. This effect is caused by the magnetic properties of $\mathrm{UO}_{2}$ and the degenerate bands giving rise to Jahn-Teller distortions. This result is unexpected since the crystal structure for both the paramagnetic and the antiferromagnetic phases of $\mathrm{UO}_{2}$ are cubic and there is no clear evidence of non-cubic distortions in diffraction experiments ${ }^{4}$. These results should also apply to the interpretation of other magnetic strongly correlated materials exhibiting Jahn-Teller physics ${ }^{5}$.

\section{Results}

Molecular dynamics simulations of $\mathrm{UO}_{2}$ thermal conductivity. Our consideration of anisotropic $\mathrm{UO}_{2}$ thermal conductivity originated from molecular dynamics (MD) simulations using the direct method $^{6-8}$, which calculates thermal conductivity by measuring the temperature gradient in a rectangular cuboid with heat added and removed at a hot and cold plate, respectively. The simulations predict large differences for the effective thermal conductivity in the $<100\rangle<110\rangle$ and $<111\rangle$ crystallographic directions at $300 \mathrm{~K}$, see Fig. 1a. In an attempt to eliminate boundary condition artifacts related to scattering by the hot and cold plates, the thermal conductivity is obtained for infinite simulation cell length by extrapolation ${ }^{7,8}$. Figure 1a shows that $\kappa^{-1}=f\left(L_{z}^{-1}\right)\left(L_{z}\right.$ is the length of the simulation cell) does not obey the expected linear relationship for $\mathrm{UO}_{2}$ in the $<100>$ direction, and is instead quadratic. The extrapolated values at $300 \mathrm{~K}$ in the inset of Fig. la (based on the quadratic fits) show that the anisotropy between $<111>$ and $<100>$ is $\sim 15 \%$. This result is noteworthy as the quadratic term indicates that the intrinsic material properties are affected, whereas a linear term would correspond to a mere change in the phonon scattering length. The underlying physics is illustrated by the projected MD trajectory of an oxygen ion, see Fig. 1b. This simulation was performed in the same rectangular cuboid cell as the thermal conductivity simulations, but without a temperature gradient. Although the average position coincides with the cubic $\mathrm{UO}_{2}$ fluorite structure, the shape of the distribution is an ellipsoid rather than the expected sphere. The ellipsoidal character is still present during short time intervals in a cubic simulation cell, however it averages to a sphere since the ellipsoid orientation along the crystallographic axes is uncorrelated in space and time. Apparently, the trajectory is sensitive to small perturbations, such as the dimensionality of the simulation cell or the applied temperature gradient. These perturbations break the underlying cubic symmetry, and lead to a preferential ellipsoid orientation along one of the three $<100>$ axes. This orientation bias gives rise to anisotropy in the physical properties.

Measurements of anisotropic $\mathrm{UO}_{2}$ thermal conductivity. These MD results inspired a detailed experimental study of the $\mathrm{UO}_{2}$ thermal conductivity as a function of temperature with a thermal gradient applied in different crystallographic directions on oriented single crystal bars. We find that the overall shape of the
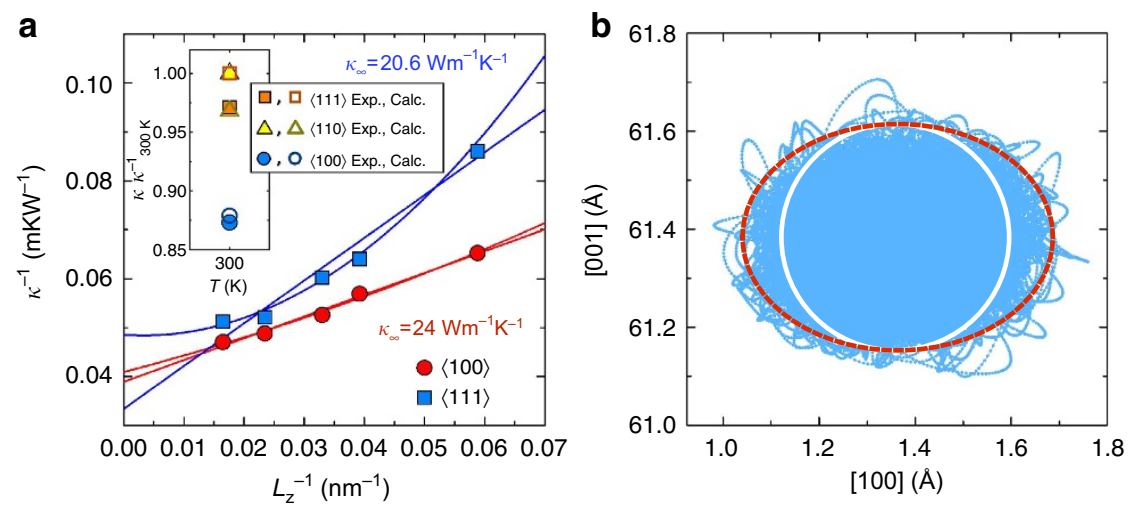

Figure 1 | Anisotropic $\mathbf{U O}_{\mathbf{2}}$ thermal conductivity from $\mathbf{M D}$ simulations. (a) The thermal conductivity $(\kappa)$ variation $\left(\kappa^{-1}\right)$ as a function of the length $\left(L_{z}^{-1}\right)$ of the simulation cell for the temperature gradient applied in the $<100>$ and $<111>$ lattice directions at $300 \mathrm{~K}$. The lines represent linear and quadratic fits to the data used for extrapolation to infinite length $\left(\kappa_{\infty}\right)$ of the simulation cells $s^{7,8}$. The inset compares the experimentally measured thermal conductivity values (Exp.) in the $<100><110>$ and $<111>$ crystallographic directions with the MD simulations (Calc.). The length of the MD simulation cells were $\sim 30 \mathrm{~nm}$ for these data points. The values have been normalized to the highest thermal conductivity value at $300 \mathrm{~K}\left(\kappa_{300}\right)$. (b) Projected trajectory of one oxygen ion during a 500 ps MD run in the rectangular cuboid simulation cell at $300 \mathrm{~K}$. The MD simulation was performed in the NVE ensemble without an applied thermal gradient. The horizontal axis is the [100] and the vertical axis the [001] direction of the fluorite lattice. The elongated direction of the simulation cell is along the vertical axis. The ellipsoid character of the distribution is emphasized by the dashed red line as compared with the white line representing the spherical distribution. 
thermal conductivity is atypical for insulating crystals without defects, see Fig. 2. For all directions, pronounced double-peak behaviour in the thermal conductivity is observed with maxima occurring at $\sim 10$ and $220 \mathrm{~K}$ and a minimum at the Néel temperature $T_{\mathrm{N}}=30.8 \mathrm{~K}$, as reported previously ${ }^{1,9}$. This behaviour has been observed in other materials with antiferromagnetic transitions ${ }^{9-13}$, which is due to resonant scattering of phonons through excitations of the spins on the magnetic ions in the paramagnetic phase and absence of this scattering mechanism below $T_{\mathrm{N}}$ due to spin ordering ${ }^{9-13}$. The temperature dependence of the $\mathrm{UO}_{2}$ thermal conductivity below $10 \mathrm{~K}$ obeys $\kappa \propto T^{1.45}$, see Fig. $2 \mathrm{~b}$, which deviates from the expected $\kappa \propto T^{3}$ dependence for phonons. Therefore we speculate that the low temperature $\mathrm{UO}_{2}$ thermal conductivity is mediated by magnons, or magnons and phonons.

Regardless of the thermal transport mechanism below $T_{\mathrm{N}}$, scattering by spin excitations on the uranium ions are responsible for the unusual shape above this temperature ${ }^{9}$, see Fig. 2 a. In particular: the maximum thermal conductivity is of low magnitude $\left(8.7 \mathrm{~W} \mathrm{~m}^{-1} \mathrm{~K}^{-1}\right)$, occurs at high temperature $(220 \mathrm{~K})$ and then decreases slowly. Typically the phonon thermal conductivity rises quickly and reaches its maximum well below $100 \mathrm{~K}$, after which phonon-phonon scattering leads to rapid decrease. In $\mathrm{UO}_{2}$, scattering by the spins on uranium ions results in a lower thermal conductivity and higher temperature for the maximum conductivity than if only phonon-phonon scattering were active. This effect is illustrated in Fig. 2c, which compares the $\mathrm{UO}_{2}$ thermal conductivity to recent measurements on $\mathrm{ThO}_{2}$ single crystals ${ }^{14}$. ThO $\mathrm{Th}_{2}$ is isostructural with $\mathrm{UO}_{2}$ although it is diamagnetic ${ }^{15}$ and thus there is no phonon-spin scattering. The maximum $\mathrm{ThO}_{2}$ thermal conductivity is $140 \mathrm{~W} \mathrm{~m}^{-1} \mathrm{~K}^{-1}$ at about $40 \mathrm{~K}^{14}$, which is a more typical case for metal oxides.

Scattering between phonons and spins on uranium ions occurs by phonons exciting the energy levels of the magnetic ions ${ }^{10-12,16-18}$. The magnitude of this resonant phonon-spin scattering mechanism is controlled by the position of these energy levels. Scattering is prominent if the excited spin states are in the same energy range as the occupied phonons, otherwise scattering is negligible ${ }^{10,16-18}$. There are no excitation levels in the triplet ground state of uranium ions above $T_{\mathrm{N}}$ that can interact with phonons ${ }^{19,20}$. However, inelastic neutron scattering experiments have identified a dynamic Jahn-Teller distortion in $<100>$ lattice directions that splits the ground state into three singlets with dispersive peaks of magnetic character between 3 and $10 \mathrm{meV}^{19,20}$. These excitations are in the appropriate energy range for interaction with phonons and give rise to significant scattering in the paramagnetic phase.

Analysis of $\mathrm{UO}_{2}$ thermal conductivity. We can gain further insight by analyzing the temperature dependence of the $\mathrm{UO}_{2}$ thermal conductivity, which above $T_{\mathrm{N}}$ can be represented by an extended Callaway model ${ }^{21}$ (see Methods). This approach applies a Debye model for the phonon spectrum and considers scattering by boundaries, point defects and Umklapp phonon-phonon processes. A Callaway model for $\mathrm{ThO}_{2}$ was modified to capture the important differences with $\mathrm{UO}_{2}$. This extension required a model for resonance spin scattering at two different energy levels $\left(\frac{1}{\tau_{S}} \propto \frac{C \omega^{4}}{\left(\omega^{2}-\omega_{S}^{2}\right)} F(T)\right.$, where $\tau_{S}$ is the spin scattering relaxation time, $\omega$ is the phonon frequency, $\omega_{S}$ is the resonance frequency, $C$ is the phonon-spin coupling constant and $F(T)$ describes the properties of the two-level system $)^{22}$. In addition to the resonance terms, the defect scattering in $\mathrm{UO}_{2}$ is increased compared with $\mathrm{ThO}_{2}$ possibly due to isotopic contributions. These fits reproduce the $\mathrm{UO}_{2}$ thermal conductivity data, see Fig. 3. The resonance energy obtained for the $<110\rangle \mathrm{UO}_{2}$ data is about $3 \mathrm{meV}$ (the two levels in the model overlap), which agrees with the lowest energy excitation measured in the inelastic neutron scattering experiments ${ }^{19,20}$. This result gives us confidence that the low $\mathrm{UO}_{2}$ thermal conductivity and its unique temperature dependence are due to resonance scattering of phonons by excitations involving spins on the uranium ions. The Callaway model parameters are found in Supplementary Table 1.

At low temperature, anisotropic thermal conductivity in cubic materials has been previously observed and attributed to ballistic
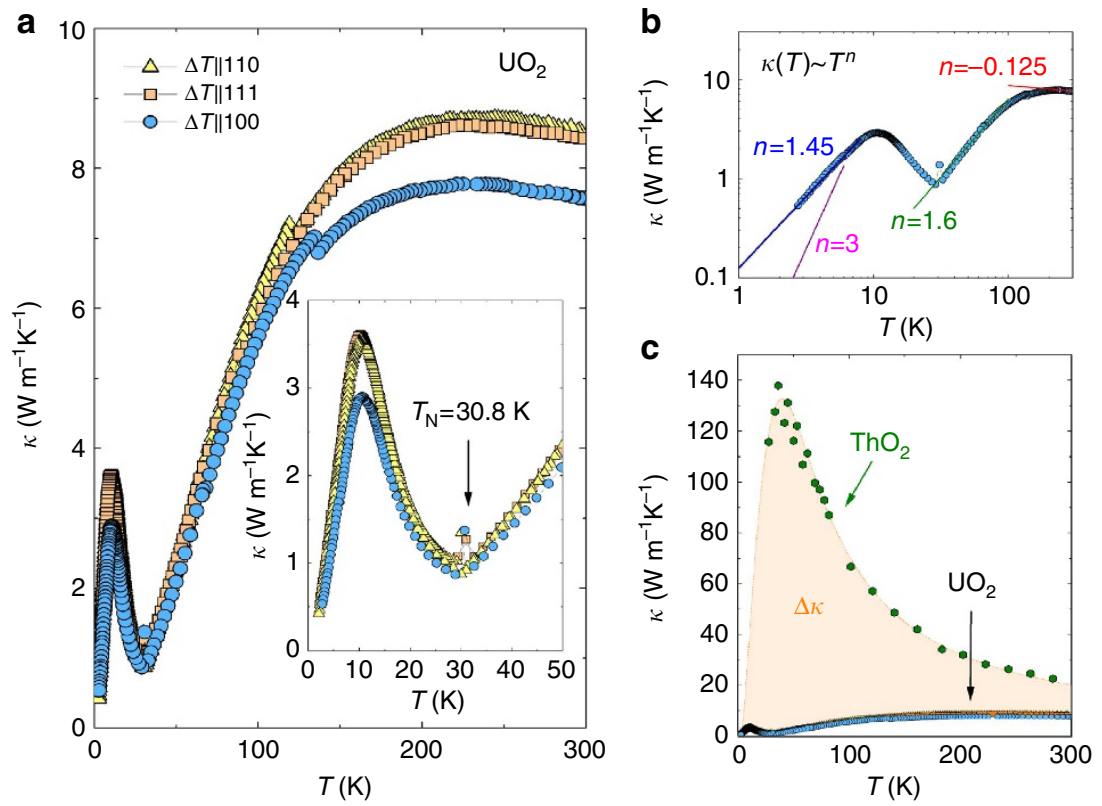

Figure 2 | Measured $\mathbf{U O}_{\mathbf{2}}$ thermal conductivity. (a) $\cup_{2}$ thermal conductivity $(\kappa)$ in the $\left.\left.<100\right\rangle<110\right\rangle$ and $<111>$ crystallographic directions (please refer to Methods for details on sample preparation and the thermal conductivity measurements). The inset highlights the thermal conductivity in the region of the Néel temperature $\left(T_{N}\right)$. The spike at $T_{N}$ is a consequence of the divergence of the heat capacity. (b) Log-log plot of thermal conductivity as function of temperature $(T)$. (c) Measured $\mathrm{UO}_{2}$ thermal conductivity compared with reference data for $\mathrm{ThO}_{2}($ ref. 14). 


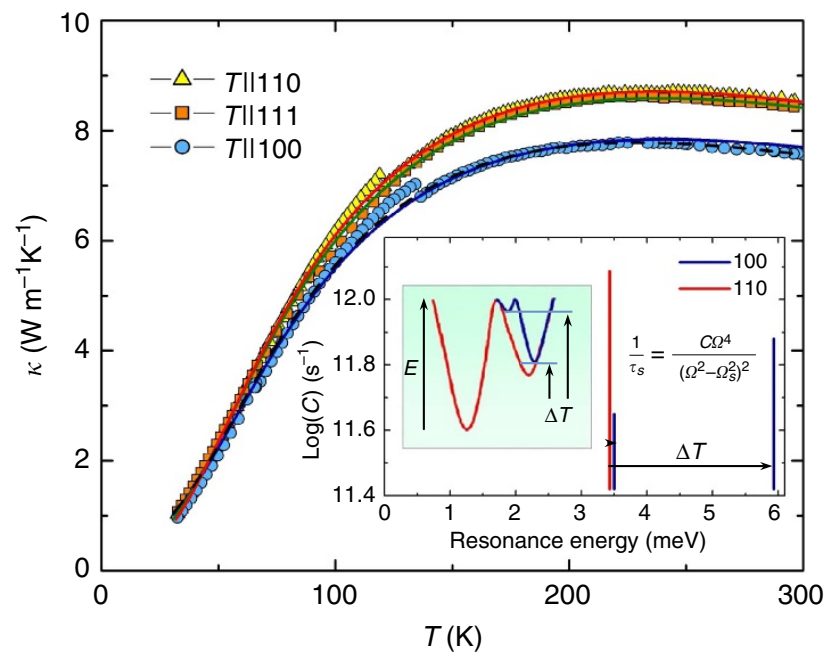

Figure 3 | Measured $\mathrm{UO}_{2}$ thermal conductivity fitted to the extended Callaway model. Markers show the measured thermal conductivity $(\kappa)$ data and lines the Callaway fits. The anisotropy is captured by shifting the energies ( $\hbar \omega_{S}$, where $\hbar$ is the reduced Planck constant and $\omega_{S}$ is the resonance frequency) and coupling constants $(C)$ in the resonance spin scattering terms $\left(\frac{1}{\tau_{s}}\right)$, see equation in the inset. The difference in resonance scattering parameters for the $<110>$ and $<100>$ crystallographic directions is illustrated in the inset. The arrows show the shifts occurring due to the applied temperature gradient $(\Delta T)$ in the $<100>$ direction. The second inset schematically illustrates the same shift and splitting in terms of potential wells $(E)$. The dashed line for $\mathrm{UO}_{2}<100>$ represents a fit where both the spin and Umklapp scattering terms were allowed to change, which differs from solid line where only the spin scattering terms were treated as free parameters.

phonon transport or phonon focusing mechanisms ${ }^{2}$. However, these explanations are not possible in $\mathrm{UO}_{2}$ because the short mean free path due to spin scattering rules out boundary scattering from macroscopic sample surfaces as the mechanism limiting phonon lifetimes. This conclusion is also supported by the anisotropy persisting at least $150 \mathrm{~K}$ above the maximum thermal conductivity. Sample impurities and oxygen nonstoichiometry were also ruled out as explanation for the anisotropy (see Methods). In addition, scattering due to the presence of different isotopes, which has important effect on other fluorite oxides (for example, $\mathrm{CeO}_{2}, \mathrm{HfO}_{2}$ and $\mathrm{ZrO}_{2}$ ), is not a significant issue in depleted uranium samples considered in this study (see Methods). $\mathrm{ThO}_{2}$ has only one naturally occurring isotope and thus behaves similar to $\mathrm{UO}_{2}$ with regards to isotope effects on thermal conductivity.

Anisotropy of $\mathrm{UO}_{2}$ thermal conductivity below $\boldsymbol{T}_{\mathrm{N}}$. Below $T_{\mathrm{N}}$ the anisotropy may not seem surprising, since Jahn-Teller distortions of the oxygen sublattice exist ${ }^{23,24}$. This reduced symmetry originates from the transverse $3 \mathbf{k}$ dipolar ordering and leads to a different space group: $F m \overline{3} m$ to $P a \overline{3}$ (ref. 25). This behaviour is driven by similar ordering of the uranium electric quadrupoles ${ }^{26}$. While, $\mathrm{Pa} \overline{3}$ has reduced symmetry with respect to $F m \overline{3} m$ it is still a cubic space group that requires that the thermal conductivity must fulfil cubic symmetry relations and cannot explain the anisotropy. Whether the thermal conductivity below $T_{\mathrm{N}}$ is due to phonons, magnons or both, we suggest that the key to understanding the anisotropy is the [ $\xi 00]$ optical magnon branches, and for phonon mechanisms, the scattering that they give rise to. Measurements of the magnon dispersion in $\mathrm{UO}_{2}$ have revealed an optical $[\xi 00]$ branch with a minimum at the [100] reciprocal lattice point of around $2 \mathrm{meV}(23.2 \mathrm{~K})$ and a maximum at [000] of $10-12 \mathrm{meV}(116.2-139.3 \mathrm{~K})$ (refs 19,20), which is the required energy range to couple with phonons below $T_{\mathrm{N}}$. The [ $\xi 00]$ magnons or the phonon-magnon scattering by themselves do not explain the observed anisotropy, since the cubic crystal symmetry dictates equal contribution from all [ $\xi 00]$ branches. However, the temperature gradient applied during the thermal conductivity measurements interacts with the magnetovibrational properties of the $\mathrm{UO}_{2}$ lattice such that the [ $\left.\xi 00\right]$ branches are affected differently when the gradient is applied in, for example, the $\langle 100\rangle\langle 110\rangle$ and $<111\rangle$ crystallographic directions. The temperature gradient alone does not give rise to this behaviour, but instead we propose that the small electric field generated by the temperature gradient is the source. This behaviour is facilitated by $\mathrm{UO}_{2}$ having a large Seebeck coefficient ${ }^{27}$. We suggest that this field interacts with the electric moments on uranium ions in $\mathrm{UO}_{2}$ to change the [ $\left.\xi 00\right]$ magnon properties. The fact that the major anisotropy occurs in the $<100\rangle$ direction coincides with the small $C_{44}$ elastic constant of $\mathrm{UO}_{2}$, the minimum of which is at $T_{\mathrm{N}}$ (ref. 28).

Anisotropy of $\mathrm{UO}_{2}$ thermal conductivity above $T_{\mathrm{N}}$. Above $T_{\mathrm{N}}$, the anisotropy is even more unexpected, since paramagnetic $\mathrm{UO}_{2}$ has always been measured to be a perfect cubic fluorite. In this temperature range phonons in the paramagnetic phase are scattered by the resonant states that originate from splitting of the triplet ground state of uranium ions into singlets by the dynamical Jahn-Teller distortions ${ }^{19}$. These lattice distortions alone do not explain the anisotropy. As for below $T_{\mathrm{N}}$, we speculate that it is caused by the applied temperature gradient interacting with the magneto-vibrational properties and giving rise to different response when applied in the $\langle 100\rangle<110\rangle$ and $<111\rangle$ crystallographic directions. This can be viewed as the gradient slightly changing the character of the distortions. This mechanism was investigated by fitting the extended Callaway model to the $<100><110>$ and $<111>$ experimental thermal conductivity results. The $<110\rangle$ model was used as a baseline and the other conductivities were then fitted by allowing the resonance scattering terms to change (see Methods). All other parameters were held fixed. The results shown in Fig. 3 reveal that the anisotropy in the $<111>$ and $<100>$ directions is accommodated by splitting and shifting the two resonance energies by 0.37 to $2.4 \mathrm{meV}$, and modifying the phonon-spin coupling constants. This situation is similar to reducing the symmetry by application of an external field $^{29}$; however, it is surprising that the small temperature gradients used in the thermal conductivity experiments have such a profound effect on the conductivity. An important feature of this model is that it explains the anisotropy of the thermal conductivity, while not requiring deviation from the cubic crystal structure measured in diffraction ${ }^{4}$. These findings are a result of the anisotropy arising from scattering processes that only develop non-cubic character in the presence of temperature gradients. The measured thermal conductivity values in the $\langle 111\rangle$ and $<110\rangle$ directions agree with recent phonon lifetime measurements $\left(8.4 \pm 1.5 \mathrm{~W} \mathrm{~m}^{-1} \mathrm{~K}^{-1}\right.$ at $295 \mathrm{~K}$ compared with our measurements of 8.53 and $8.44 \mathrm{~W} \mathrm{~m}^{-1} \mathrm{~K}^{-1}$ for the $<110>$ and $\langle 111\rangle$ directions, respectively $)^{30}$. Since lifetime measurements do not apply temperature gradients, it follows that the best agreement occurs for the $\langle 111\rangle$ and $\langle 110\rangle$ directions. These two directions do not couple to the applied temperature gradient, in contrast to the $<100>$ direction for which the corresponding thermal conductivity is $7.59 \mathrm{~W} \mathrm{~m}^{-1} \mathrm{~K}^{-1}$. The Callaway model for the different crystallographic directions can be used to extrapolate the thermal conductivities, which shows that the anisotropy remains even at high temperature, for example, it is predicted 
to be $\sim 10 \%$ at $1,200 \mathrm{~K}$. The small discontinuity in the thermal conductivity between 100 and $150 \mathrm{~K}$ coincides with the onset of anisotropy. The source of the discontinuity is still under investigation.

\section{Discussion}

Returning to the MD simulations, the phonon-spin scattering origin of the anisotropy cannot be captured since the empirical potential does not account for magnetism. Furthermore, the lack of explicit phonon-spin scattering models results in an overestimation of thermal conductivity compared with experiments, see Figs 1 and 2. At $300 \mathrm{~K}$, the simulated thermal conductivity values for $\mathrm{UO}_{2}$ are closer to the experimental results for $\mathrm{ThO}_{2}$ (no phonon-spin scattering) than to the $\mathrm{UO}_{2}$ data. Nevertheless the fact that the empirical potential still captures the anisotropic response of $\mathrm{UO}_{2}$ in a temperature gradient (see inset of Fig. 1a), suggests that some of the physics arising from the phonon-spin scattering has been embedded into the force field description via the soft $\mathrm{C}_{44}$ elastic constant and lattice anharmonicity ${ }^{31}$.

In summary, the results of this study show that an applied temperature gradient interacts with the electric moments on uranium ions to slightly break cubic symmetry and leads to anisotropy in physical properties. Specifically, the measured and calculated anisotropic $\mathrm{UO}_{2}$ thermal conductivity give clear evidence of $\mathrm{UO}_{2}$ exhibiting properties of a non-cubic compound, because second-rank tensors, such as thermal conductivity, must result in isotropic behaviour for cubic materials. This result is unexpected since the crystal structure for the paramagnetic as well as the antiferromagnetic phases of $\mathrm{UO}_{2}$ are cubic and there is no clear evidence of non-cubic distortions in diffraction experiments.

\section{Methods}

MD simulations. MD simulations applying the direct method ${ }^{6,7}$ were used to calculate $\mathrm{UO}_{2}$ thermal conductivity. All calculations were performed with the scalable parallel short-range MD code ${ }^{32}$ and closely follow the study by Watanabe et al. ${ }^{8}$ A constant heat flow was imposed in a rectangular cuboid simulation cell by allowing thermal energy to transmit from a hot plate to a cold plate separated by half of the simulation cell length. The thickness of the hot and cold plates was $5.463 \AA$, that is, one $\mathrm{UO}_{2}$ unit cell. The heat flow was set to $0.05 k_{\mathrm{B}} T / A$ ( $k_{\mathrm{B}}$ is the Boltzmann constant and $T$ is the temperature) per time step, where $A$ is the cross section area of the simulation cell. Periodic boundary conditions were applied to the simulation cell. The size of the time step was $1.0 \mathrm{fs}$ in all simulations. To equilibrate the thermal and strain properties of the system, each simulation was initiated by performing constant temperature and pressure simulations for $12.5 \mathrm{ps}$. The equilibration time after setting up the heat flow was $500 \mathrm{ps}$, and $500-1,000 \mathrm{ps}$ were used as the data acquisition time. The temperature gradient resulting from the heat flux was monitored and averaged over the acquisition time to obtain the thermal conductivity via Fourier's law, $J_{z}=-\kappa_{z} \frac{d T}{d z}$, where $J_{z}$ is the imposed heat flux, $z$ represents the crystallographic direction of the temperature gradient and $\kappa_{z}$ is the effective conductivity in this direction.

The inter-atomic forces between uranium and oxygen atoms were described by the short-range potential according to the study by Basak et al. ${ }^{31}$, and the longrange electrostatic interactions were calculated by the Wolf summation method ${ }^{33}$. The length of simulation cells ranged from 16 to $60 \mathrm{~nm}$ for the simulations along the $\langle 100\rangle\langle 110\rangle$ and $\langle 111\rangle$ crystallographic directions. These relatively short cells suffer from significant phonon scattering from the hot and cold plates, especially at low temperature. This is an artifact of the simulation setup, but the small cells are nevertheless useful for establishing physical trends and the results for infinite cell length can be obtained by extrapolation of $\frac{1}{\kappa}=\frac{1}{\kappa_{\infty}}+\frac{c}{L_{z}}$ to $\frac{1}{L_{z}}=0$ (refs 7,8 ), where $c$ is a constant related to scattering by the hot and cold plates, $L_{z}$ is the length of the simulation cell and $\kappa_{\infty}$ is the thermal conductivity for a simulation cell of infinite length. Due to the non-linear effects in the $<100>$ direction originating from the ellipsoidal character of the projected oxygen trajectory shown in Fig. 1b, the following quadratic $\left(\frac{c_{2}}{L_{2}^{2}}\right)$ relation was also used for extrapolation $\frac{1}{\kappa}=\frac{1}{\kappa_{\infty}}+\frac{c_{1}}{L_{z}}+\frac{c_{2}}{L_{z}^{2}}$, where $c_{1}$ and $c_{2}$ are constants. As for the linear model, $c_{1}$ captures the scattering from the hot and cold plates, while $c_{2}$ is related to the $\mathrm{UO}_{2}$ lattice properties, which includes the ellipsoidal behavior of the oxygen trajectory in Fig. 1b. In the directions perpendicular to the thermal current, the simulation cells have lengths ranging from 3.7 to $4.0 \mathrm{~nm}$. The dependence of the computed thermal conductivity on cross-sectional area has been shown to be weak ${ }^{34}$. However, for $\mathrm{UO}_{2}$, the dimensions of the simulation cell impact the ellipsoidal character of the projected oxygen trajectory illustrated in Fig. 1b. If the simulation in Fig. 1b is carried out in a cubic cell, the shape of the corresponding trajectory is spherical, which is expected for perfectly cubic or isotropic materials. This implies that the cuboid simulation cell slightly, but significantly, perturbs the $\mathrm{UO}_{2}$ lattice properties, which is likely related to the soft $C_{44}$ elastic constant ${ }^{28}$. Ultimately, this perturbation results in anisotropic thermal conductivity. Ballistic phonon transport, which occurs if scattering of phonons in the simulation cell is minimal, is another possible explanation for the anisotropy. This is because under ballistic conditions, thermal conductivity is a fourth rank tensor related to the elastic stiffness tensor and anisotropic thermal conductivity could arise in cubic materials ${ }^{2,3}$. In the MD simulations, ballistic transport is intertwined with the dependence of thermal conductivity on the simulation cell length and scattering by the hot and cold plates. The extrapolation scheme should eliminate ballistic contributions, however it cannot be conclusively ruled out based on the present simulations. The results obtained from the scalable parallel short-range MD code in this study were also confirmed using the LAMMPS MD code ${ }^{35}$.

Thermal conductivity measurements. The depleted $\mathrm{UO}_{2}$ single crystals used for the thermal conductivity measurements were obtained by the Los Alamos Scientific Laboratory (LASL, now Los Alamos National Laboratory-LANL) from the Battelle Northwest Laboratory in 1969. Samples were prepared by fusing depleted PWRgrade $\mathrm{UO}_{2}$ powder in an arc furnace and large crystals were harvested from the bulk $^{36}$. Before delivery to LASL, crystals were annealed in moist hydrogen to remove inclusions and assure stoichiometry. We performed thermal gravimetric analysis on the samples studied and the results confirmed the original assertion of stoichiometry to be $<2.001$ (original coulometric titration in 1969 resulted in a stoichiometry of 2.0006). Regarding chemical purity, analysis performed at the time of original synthesis confirmed high purity samples $(>99.9688 \%)$. By emission spectrographic analysis Ag, B, Fe, Sn and so on were below 200 ppm total, C below 50 ppm, Cl less than $10 \mathrm{ppm}$ and $\mathrm{F}$ less than $2 \mathrm{ppm}$. The total nitrogen content was found to be $50 \mathrm{ppm}$ as determined by Kjeldahl analysis. The isotopic purity of the samples was ${ }^{238} \mathrm{U}>99.78 \%$ (the balance is ${ }^{235} \mathrm{U}$ ). Optical microscopy showed the samples to be free of macroscopic defects (cracks and so on). Oriented rectangular cuboids were prepared from a large single crystal, see Supplementary Fig. 1 for an example of the crystal bars, and orientations were verified by standard Laue back-reflection X-ray technique. As an example Supplementary Fig. 2 shows a typical Laue diffraction pattern for $\langle 111\rangle$ samples. The character of the pattern highlights the high quality of the crystal. The misalignment is estimated to be $<0.5$ degrees for all samples. In addition, we prepared a $<110>$ sample that was misaligned by 8 degrees. The thermal conductivity of the second $<110\rangle$ crystal was almost identical to the first sample. The close agreement between the two $<110>$ crystals also confirms that the original single crystal was homogeneous, and thus our results of anisotropic thermal conductivity cannot be explained by variations in the $\mathrm{UO}_{2}$ crystal properties between the oriented samples. This is further evidenced by the fact that the difference in the thermal conductivities between the $\langle 110\rangle\langle 111\rangle$ and $<100>$ samples approaches zero at the Néel temperature. X-ray diffraction was also performed on all of the single crystal samples, which confirmed the fluorite structure and lattice parameters consistent with literature data.

The $\mathrm{UO}_{2}$ thermal conductivity measurements were performed in a Physical Property Measurement System-9 from Quantum Design equipped with a $9 \mathrm{~T}$ superconducting magnet and using samples with typical dimensions of $\sim 1 \times 1 \times 7 \mathrm{~mm}$. Both four-terminal and two-terminal probe lead configurations were used to measure thermal conductivity. A silver-filled H20E epoxy was used to connect the sample to the leads, which are gold-coated copper. We used the continuous mode $\left(0.1 \mathrm{Kmin}^{-1}\right)$ in the temperature range $4-300 \mathrm{~K}$. To gain down the bridge, it is important to use $40 \mathrm{mV}$ as the upper power limit because the resistivity of the sample is in the kOhm regime (at room temperature). According the Quantum Design handbook the typical accuracy, taking into account radiation losses, of thermal conductivity measurements using a TTO option is $\pm 5 \%$. However, since our measurements are performed on the same material, the relative accuracy between the oriented samples is much higher. To ensure that the sample mounting, for example, the leads attached to the ends of the $\mathrm{UO}_{2}$ samples, did not bias the results, we demounted the $\langle 100\rangle$ and $\langle 110\rangle$ samples, annealed them in dry $\mathrm{Ar}-6 \% \mathrm{H}_{2}$ gas at $850^{\circ} \mathrm{C}$ for 18 hours to ensure sample stoichiometry, remounted the sample and repeated the thermal conductivity measurements, which reproduced the original results. The variation was well within the uncertainty range of the methodology, which confirms the validity of the anisotropic thermal conductivity. The second measurement series used two instead of four wire terminals. The $<110\rangle$ and $<111>$ results agree with earlier single crystal measurements ${ }^{2}$ and they are thus deemed to be accurate. We reiterate that any defects differentiating the $\langle 110\rangle<111\rangle$ and $\langle 100\rangle$ samples would result in a constant shift in the thermal conductivities, which is not observed in our measurements, see Fig. 2. A final argument against sample defects or concerns about the sample mounting is obtained by comparing the anisotropy at, for example, the maximum of the low temperature thermal conductivity peak with the anisotropy for the same magnitude of the conductivity at the second peak. The anisotropy is not the same, which should be the case if defects or inaccurate sample mounting were responsible for the measured anisotropy. From this we conclude that the anisotropic thermal conductivity cannot be explained by simple defects or measurement uncertainties.

The Callaway model. Above $T_{\mathrm{N}}$ the measured thermal conductivities were analyzed in terms of the Callaway model ${ }^{21}$, which assumes a Debye phonon spectrum 
and expresses thermal conductivity as:

$$
\kappa=\frac{k_{\mathrm{B}}}{2 \pi v}\left(\frac{k_{\mathrm{B}} T}{\hbar}\right)^{3} \int_{0}^{\Theta_{\mathrm{D}} / T} \frac{\tau_{\mathrm{p}} x^{4} e^{x}}{\left(e^{x}-1\right)^{2}} d x
$$

where $v$ is the mean velocity of sound, $\tau_{\mathrm{p}}$ stands for the phonon relaxation time, $x=\hbar \omega / k_{\mathrm{B}} T, \omega$ is the phonon frequency, while $\hbar, \Theta_{\mathrm{D}}$ and $k_{\mathrm{B}}$ are the reduced Planck constant, the Debye temperature and the Boltzmann constant, respectively. The mean sound velocity was taken as $v=3,171 \mathrm{~ms}^{-1}$ for $\mathrm{UO}_{2}$, which was calculated from $v=k_{\mathrm{B}} \Theta_{\mathrm{D}} / \hbar \sqrt[3]{6 \pi^{2} n}\left(\Theta_{\mathrm{D}}=395 \mathrm{~K}^{37}\right)$, where $n$ is the number of atoms per unit volume. The relaxation time may be accounted for by considering possible scattering mechanisms for heat transport. This typically includes point defect scattering $(D)$, boundary scattering $(B)$, Umklapp processes $(U)$ and spin scattering $(S), \tau_{\mathrm{p}}^{-1}=\tau_{D}^{-1}+\tau_{B}^{-1}+\tau_{U}^{-1}+\tau_{S}^{-1}$, where the particular inverse relaxation times are given by the following expressions ( $D, B$ and $U$ are constants fitted to thermal conductivity data):

$$
\begin{gathered}
\tau_{D}^{-1}=D x^{4} T^{4}=D\left(\frac{\hbar \omega}{k_{\mathrm{B}}}\right)^{4} \\
\tau_{B}^{-1}=B \\
\tau_{U}^{-1}=U T^{3} x^{2} e^{-\Theta_{\mathrm{D}} / b T}=U T\left(\frac{\hbar \omega}{k_{\mathrm{B}}}\right)^{2} e^{-\Theta_{\mathrm{D}} / b T} \\
\tau_{S}^{-1}=\sum_{i} \frac{C_{i} \omega^{4}}{\left(\omega^{2}-\omega_{S, i}^{2}\right)} F_{i}(T)
\end{gathered}
$$

The spin scattering term ${ }^{22}$ is an extension to the standard Callaway model, which is required for paramagnetic $\mathrm{UO}_{2}$. In the expression for $\tau_{S}^{-1}, \omega_{S, i}$ is the resonance frequency, $C_{i}$ the phonon-spin coupling constant and $F_{i}(T)$ contains information about the two-level system. We included two levels in our model $(i=1,2)$. Both the resonance frequencies and coupling constants were treated as free parameters. The $b$ parameter in the Umklapp expression is here treated as a fitting parameter, but it may also be set to a constant $(b=2)$.

To have a realistic starting point for the free parameters in the $\mathrm{UO}_{2}$ model, we used the Callaway model parameters derived for $\mathrm{ThO}_{2}$ from the data reported in ref. 14. The mean sound velocity for $\mathrm{ThO}_{2}$ was calculated to be $2,129 \mathrm{~m} \mathrm{~s}^{-1}$ for $\Theta_{\mathrm{D}}=259 \mathrm{~K}^{38}$. The $\mathrm{ThO}_{2}$ results are listed in Supplementary Table 1 and compared with the experimental data in Supplementary Fig. 3. For $\mathrm{UO}_{2}$, we first fit the $<110>$ data by a Callaway model that does not include the phonon-spin scattering contribution $\left(\mathrm{UO}_{2}<110>\mathrm{B}\right.$ in Supplementary Table 1 and Supplementary Fig. 3). The results are somewhat unusual on initial inspection; in particular the mean free path for boundary scattering is extremely low $(7.3 \mathrm{~nm})$. The boundary term is many orders of magnitude smaller than the dimensions of the sample. This can be explained by phonon-spin scattering, which is not explicitly included in the standard Callaway model, but rather captured by the boundary term. Note that the boundary scattering term for $\mathrm{ThO}_{2}$ gives a mean free path of $8.0 \mu \mathrm{m}$, which is more than three orders of magnitude larger than for $\mathrm{UO}_{2}$ $(7.3 \mathrm{~nm})$, as explained by the lack of phonon-spin scattering in $\mathrm{ThO}_{2}$. The fact that the defect scattering terms are similar between $\mathrm{UO}_{2}$ and $\mathrm{ThO}_{2}$, see Supplementary Table 1, indicates that isotopic scattering is not responsible for the difference in thermal conductivity. Although the $\mathrm{UO}_{2}$ samples are not completely isotopically pure $(99.78 \%)$ and the $\mathrm{ThO}_{2}$ samples are, the high mass of $\mathrm{U}$ atoms means that isotope scattering is small in comparison to phonon-spin scattering. Even though the quality of the $\mathrm{UO}_{2}$ fits obtained from the standard Callaway model is good, the physical insights that can be gained from these results are limited.

In the next step, we added the spin scattering terms to the Callaway model, which we label the extended Callaway model, and fitted the $\mathrm{UO}_{2}<110>$ thermal conductivity data, see Supplementary Table 1 and Fig. 3, starting from the $\mathrm{ThO}_{2}$ parameters. The reduced $\mathrm{UO}_{2}$ thermal conductivity compared with $\mathrm{ThO}_{2}$ is captured by the spin scattering terms and a decrease in the defect scattering length. The boundary scattering term remains similar to the $\mathrm{ThO}_{2}$ case. The two resonance frequencies essentially overlap for the $<110>$ fit. The $<111>$ and $<100>\mathrm{UO}_{2}$ thermal conductivity data was then fitted by only allowing the spin scattering terms to change, keeping all other parameters fixed at the $\langle 110\rangle$ values, see Supplementary Table 1 and Fig. 3. The quality of the $<111>$ fit is excellent and for $\langle 100\rangle$ it is also good, even though not perfect. For $<100\rangle$ a better fit is easily obtained by allowing the Umklapp parameters to change, see Supplementary Table 1 and Fig. 3. Compared with the extended Callaway model for the $<110>$ conductivity, the $\langle 111\rangle$ and $\langle 100\rangle$ anisotropies are accommodated by shifting and splitting the spin resonance frequencies or energies and slightly modifying the phonon-spin coupling terms. As discussed in Results, we propose that these changes are induced by the temperature gradient applied in the thermal conductivity experiments.

\section{References}

1. Haase, V. et al. Gmelin Handbook of Inorganic Chemistry (Springer Verlag, 1986).

2. McCurdy, A., Maris, H. \& Elbaum, C. Anisotropic heat conduction in cubic crystals in the boundary scattering regime. Phys. Rev. B 2, 4077-4083 (1970).
3. Northrop, G. A. \& Wolfe, J. P. Ballistic phonon imaging in germanium. Phys. Rev. B 22, 6196-6212 (1980).

4. Willis, B. T. M. Neutron diffraction studies of the actinide oxides. I. Uranium dioxide and thorium dioxide at room temperature. Proc. Roy. Soc. Lond. A 274, 122 (1963)

5. Santini, P. et al. Multipolar interactions in f-electron systems: the paradigm of actinide dioxides. Rev. Mod. Phys. 81, 807-863 (2009).

6. Jund, P. \& Jullien, R. Molecular-dynamics calculation of the thermal conductivity of vitreous silica. Phys. Rev. B 59, 13707-13711 (1999).

7. Schelling, P. K., Phillpot, S. R. \& Keblinski, P. Comparison of atomic-level simulation methods for computing thermal conductivity. Phys. Rev. B 65, 144306 (2002).

8. Watanabe, T. et al. Thermal transport properties of uranium dioxide by molecular dynamics simulations. J. Nucl. Mater. 375, 388-396 (2008).

9. Moore, J. \& McElroy, D. Thermal conductivity of nearly stoichiometric singlecrystal and polycrystalline $\mathrm{UO}_{2}$. J. Am. Ceram. Soc. 54, 40-46 (1971).

10. Slack, G. Thermal conductivity of $\mathrm{CaF}_{2}, \mathrm{MnF}_{2}, \mathrm{CoF}_{2}$, and $\mathrm{ZnF}_{2}$ crystals. Phys Rev. 122, 1451-1462 (1961).

11. Hofmann, M. et al. Strong damping of phononic heat current by magnetic excitations in $\mathrm{SrCu}_{2}\left(\mathrm{BO}_{3}\right)_{2}$. Phys. Rev. Lett. 87, 047202 (2001).

12. Sales, B. C., Lumsden, M. D., Nagler, S. E., Mandrus, D. \& Jin, R. Magnetic field enhancement of heat transport in the $2 \mathrm{~d}$ heisenberg antiferromagnet $\mathrm{K}_{2} \mathrm{~V}_{3} \mathrm{O}_{8}$. Phys. Rev. Lett. 88, 095901 (2002).

13. Hess, C. et al. Magnon heat transport in doped $\mathrm{La}_{2} \mathrm{CuO}_{4}$. Phys. Rev. Lett. 90, 197002 (2003).

14. Mann, M. et al. Hydrothermal growth and thermal property characterization of $\mathrm{ThO}_{2}$ single crystals. Cryst. Growth Des. 10, 2146-2151 (2010).

15. Jain, M. \& Gupta, A. Diamagnetic Susceptibility and Anisotropy of Inorganic and Organometallic Compounds, Landolt-Börnstein-Group II Molecules and Radicals Vol. 27A (Springer Verlag, 2007).

16. Van Vleck, J. H. Paramagnetic relaxation times for titanium and chrome alum. Phys. Rev. 57, 426-447 (1940).

17. Slack, G. \& Galginaitis, S. Thermal conductivity and phonon scattering by magnetic impurities in CdTe. Phys. Rev. 133, A253-A268 (1964).

18. Mattuck, R. D. \& Strandberg, M. W. P. Spin-phonon interaction in paramagnetic crystals. Phys. Rev. 119, 1204-1217 (1960).

19. Caciuffo, R. et al. Magnetic excitations and dynamical jahn-teller distortions in $\mathrm{UO}_{2}$. Phys. Rev. B 59, 13892 (1999).

20. Caciuffo, R. et al. Multipolar, magnetic, and vibrational lattice dynamics in the low-temperature phase of uranium dioxide. Phys. Rev. B 84, 104409 (2011).

21. Callaway, J. Model for lattice thermal conductivity at low temperatures. Phys. Rev 113, 1046-1051 (1959)

22. Neelmani \& Verma, G. S. Phonon conductivity of trivalent rare-earth-doped gallium and aluminium garnets. Phys. Rev. B 6, 3509-3515 (1972).

23. Faber, J., Lander, G. \& Cooper, B. Neutron-diffraction study of $\mathrm{UO}_{2}$ : observation of an internal distortion. Phys. Rev. Lett. 35, 1770-1773 (1975)

24. Faber, J. \& Lander, G. Neutron diffraction study of $\mathrm{UO}_{2}$ : antiferromagnetic state. Phys. Rev. B 14, 1151-1164 (1976).

25. Burlet, P. et al. Neutron diffraction on actinides. J. Less Common Met 121, 121-139 (1986).

26. Wilkins, S. et al. Direct observation of electric-quadrupolar order in $\mathrm{UO}_{2}$. Phys. Rev. B 73, 060406(R) (2006).

27. Ruello, P., Petot-Ervas, G., Petot, C. \& Desgranges, L. Electrical conductivity and thermoelectric power of uranium dioxide. J. Am. Ceram. Soc. 88, 604-611 (2005)

28. Brandt, O. G. \& Walker, C. T. Temperature dependence of elastic constants and thermal expansion for $\mathrm{UO}_{2}$. Phys. Rev. Lett. 18, 11-13 (1967).

29. Challis, L. J., McConachie, M. A. \& Williams, D. J. Magnetoresistance measurements paramagnetic ions in magnesium oxide by thermal magnetoresistance measurements. Proc. R. Soc. Lond. A 310, 493-524 (1969).

30. Pang, J. W. L. et al. Phonon lifetime investigation of anharmonicity and thermal conductivity of $\mathrm{UO}_{2}$ by neutron scattering and theory. Phys. Rev. Lett. 110, 157401 (2013)

31. Basak, C., Sengupta, A. \& Kamath, H. Classical molecular dynamics simulation of $\mathrm{UO}_{2}$ to predict thermophysical properties. J. Alloys Compd. 360, 210-216 (2003).

32. Kadau, K., Germann, T. C. \& Lomdahl, P. S. Molecular dynamics comes of age: 320 billion atom simulation on Bluegene/L. Int. J. Mod. Phys. C 17, 1755-1761 (2006).

33. Wolf, D., Keblinski, P., Phillpot, S. R. \& Eggebrecht, J. Exact method for the simulation of Coulombic systems by spherically truncated, pairwise $r^{-1}$ summation. J. Chem. Phys. 110, 8254-8282 (1999).

34. Zhou, X. W., Aubry, S., Jones, R. E., Greenstein, A. \& Schelling, P. K. Towards more accurate molecular dynamics calculation of thermal conductivity: case study of GaN bulk crystals. Phys. Rev. B 79, 115201 (2009).

35. Plimpton, S. Fast parallel algorithms for short-range molecular dynamics. J. Comput. Phys. 117, 1-19 (1995).

36. Ellis, W. \& Schwoebel, R. LEED from surface steps on $\mathrm{UO}_{2}$ single crystals. Surf. Sci. 11, 82-98 (1968) 
37. Dolling, G., Cowley, R. \& Woods, A. The crystal dynamics of uranium dioxide. Can. J. Phys. 43, 1397-1413 (1965).

38. Ali, M. \& Nagels, P. Evaluation of the Debye temperature of thorium dioxide. Phys. Status Solidi 21, 113-116 (1967).

\section{Acknowledgements}

We acknowledge Mark T. Paffett for making the $\mathrm{UO}_{2}$ single crystal available to us and Walt Ellis and Harlan Anderson for providing the original crystals. Gerry H. Lander is acknowledged for fruitful discussions and advice. This work was sponsored by the US Department of Energy, the Office of Nuclear Energy, the Nuclear Energy Advanced Modeling and Simulation (NEAMS) program. The Los Alamos National Laboratory, an affirmative action/equal opportunity employer, is operated by the Los Alamos National Security, LLC, for the National Nuclear Security Administration of the US Department of Energy under Contract No. DE-AC52-06NA25396.

\section{Author contributions}

K.G., C.R.S. and D.A.A. initiated this work. S.D. and X.-Y.L performed molecular dynamics simulations that were designed and directed by C.R.S., B.P.U. and D.A.A. K.G.,
J.C.L., R.K.S., D.J.S. and D.D.B. prepared the single crystal samples. D.D.B. and K.J.M. ensured stoichiometry of the sample via thermogravimetric and B.L.S. characterized the samples via X-ray diffraction. Thermal conductivity measurements were performed by K.G. and J.C.L., and J.L.S. assisted in the analysis of the results. C.R.S. and D.A.A. designed and directed the research and D.A.A. led the writing of the paper with contributions from C.R.S., J.C.L., R.K.S., K.J.M., X.-Y.L., B.P.U. and K.G.

\section{Additional information}

Supplementary Information accompanies this paper at http://www.nature.com/ naturecommunications

Competing financial interests: The authors declare no competing financial interests.

Reprints and permission information is available online at http://npg.nature.com/ reprintsandpermissions/

How to cite this article: Gofryk, K. et al. Anisotropic thermal conductivity in uranium dioxide. Nat. Commun. 5:4551 doi: 10.1038/ncomms5551 (2014). 